\title{
MIR432 Pre-miRNA
}

National Cancer Institute

\section{Source}

National Cancer Institute. MIR432 Pre-miRNA. NCI Thesaurus. Code C82831.

MIR432 is an oligoribonucleotide that is encoded by the human MIR432 gene and has a role in the regulation of gene expression. 